\title{
Réflexions sur la politique de communication de la commission européenne
}

Éric Dacheux

\section{(2) OpenEdition}

1 Journals

Édition électronique

URL : http://journals.openedition.org/communicationorganisation/2323

DOI : 10.4000/communicationorganisation.2323

ISSN : $1775-3546$

Éditeur

Presses universitaires de Bordeaux

Édition imprimée

Date de publication : 1 mai 2000

ISSN : 1168-5549

Référence électronique

Éric Dacheux, «Réflexions sur la politique de communication de la commission européenne », Communication et organisation [En ligne], 17 | 2000, mis en ligne le 27 mars 2012, consulté le 19 avril 2019. URL : http://journals.openedition.org/communicationorganisation/2323 ; DOI : 10.4000/ communicationorganisation.2323

Ce document a été généré automatiquement le 19 avril 2019

(c) Presses universitaires de Bordeaux 


\title{
Réflexions sur la politique de communication de la commission européenne
}

\author{
Éric Dacheux
}

1 Les dernières élections du Parlement européen, le 13 juin 1999, ont été davantage marquées par le fort taux d'abstention que par la victoire du camp conservateur. En effet si, en France, le taux d'abstention fut de 53,24\%, il atteignit près de $70 \%$ en GrandeBretagne et aux Pays-Bas. Cette désaffection ostensible des électeurs européens n'est pas le premier signe d'un euroscepticisme galopant. En effet, le refus des Norvégiens et des Suisses de participer à l'Union européenne en 1992, ainsi que le premier non Danois au référendum de Maastricht en 1993 étaient déjà des signaux forts d'une méfiance importante d'une grande partie de la population européenne vis-à-vis de la construction de l'Europe. Cette méfiance peut s'expliquer de différentes manières: rejet de la bureaucratie tatillonne de Bruxelles, peur de voir l'identité nationale se dissoudre dans une identité européenne aux contours flous, repli généralisé sur la sphère privée, etc. Parmi toutes ces explications, il en est une régulièrement mise en avant par les députés européens (Parlement européen, 1993 ; rapport Pex, 1998) : l'insuffisance de la politique de communication mise en place par la Commission européenne. L'objet de ce texte est de mettre en lumière la part de vérité et de fausse évidence contenue dans cette explication récurrente. Pour ce faire, nous allons commencer par étudier de manière critique la politique de communication de la Commission.

\section{La politique de communication de la Commission}

2 La Commission européenne n'a pas le monopole de la communication des instances européennes. Ces dernières (Parlement européen, Conseil économique et social, etc.) ont toutes un service de communication. Cependant, puisque la Commission est l'organe d'exécution des institutions européennes, elle joue un rôle central dans la politique d'information et de communication de l'Union. La Commission est divisée en directions 
générales, sorte de ministères européens, qui possèdent chacune leur(s) responsable(s) en communication chargé(s) de mettre en œuvre des campagnes spécifiques : un CD Rom sur les transports pour la DG VII, l'organisation de «L'information Society Day Europe » pour la DG XIII, etc. Cependant, jusqu'à l'arrivée de Romano Prodi, en juin $1999^{1}$, deux entités jouaient un rôle clef dans la communication de la Commission : le Service du porte parole (SPP) et la Direction générale "Audiovisuel, information, communication culture » (DG $\mathrm{X})$. Le premier est un service de relation avec la presse qui, depuis le Traité de Rome (mars 1957), est directement attaché à la présidence de la Commission. Il organise les conférences de presse avec les journalistes accrédités. La DG X, elle, est chargée d'organiser et de coordonner la politique d'information et de communication de la Commission. C'est cette politique que nous allons maintenant expliciter.

3 La DG X gère un budget d'environ 110 millions d'euros ${ }^{2}$. Elle est composée de 377 personnes qui travaillent au sein de services horizontaux ou de directions spécialisées (audiovisuelle, culture, etc.). La DG X gère et développe des réseaux institutionnels chargés de faire descendre l'information européenne et de remonter un certain nombre de données: 3 Grands centres d'information sur l'Europe (Paris, Lisbonne, Berlin), 65 Infos Points Europe (IPE) à destination du grand public, 95 Carrefours ruraux à destination du monde agricole et 550 centres de documentation européenne (CDE) à l'usage des étudiants et chercheurs européens. Ces réseaux diffusent des brochures, dépliants, cartes, livres, etc. publiés par l'office des publications européennes du Luxembourg ${ }^{3}$, mais dont beaucoup sont réalisés par une unité spécialisée (baptisée X-D-4) de la DG X. En plus de cette politique de diffusion de l'information papier, la DG X organise de nombreux colloques et conférences, en prenant appuis, notamment, sur les 1400 universitaires du réseau des professeurs Jean Monnet ${ }^{4}$. Elle mène d'autres opérations de relations publiques comme la tenue de stands dans des foires expositions et la visite organisée de la Commission. La DG X s'est également lancée dans la communication électronique avec la réalisation de nombreux CD Rom et la création du serveur Europa. Ce dernier présente les traités européens, publie les documents écrits réalisés par la DGX, annonce les grands événements européens et offre de nombreux liens avec les différents organes européens (Eurostat, Comité des régions, etc.). Par ailleurs, la DG X possède un service de production audiovisuelle qui diffuse ses réalisations aux professionnels à travers le service télévisuel «Europe By Satellite » et produit des documentaires grand public qu'elle propose aux médias et, en particuliers, à la chaîne européenne Euronews. Enfin et surtout, dans le cadre du programme Prince (programme d'information du citoyen européen) voté par le Parlement européen, la DGX coordonne des grandes campagnes de communication qui ont lieu à l'échelle européenne comme «L'Euro, une monnaie pour l'Europe » (budget: 38 millions d'Euros) et, pour cela, fait appel à des partenaires externes (publicitaires, consultants, etc.). Force est de constater que cet ensemble de moyens n'a pas pu endiguer l'euroscepticisme. Pourquoi? Les premières explications qui viennent à l'esprit ont trait aux erreurs manifestes de la Commission en matière de communication politique.

\section{Une stratégie de communication inadaptée}

4 La Commission n'use pas forcément des outils les plus adaptés à une communication politique à l'échelle européenne. Par exemple, elle a longtemps pensé que la télévision avait le pouvoir symbolique de créer le sentiment d'appartenance à une communauté. 
Dans les années quatre-vingt, les instances européennes étaient persuadées que, grâce à la télévision sans frontière, « un plus grand nombre de téléspectateurs en Europe aurait la possibilité de voir des programmes télévisés d'autres pays ou des programmes télévisés européens ce qui pourrait contribuer à la compréhension entre les peuples, améliorer la connaissance de la culture et de l'histoire d'autres peuples ainsi que promouvoir la formation d'une conscience européenne" (Commission européenne, 1984, p. 32). Aujourd'hui, l'arrivée massive des chaînes thématiques, le relatif insuccès d'Euronews et les difficiles cohabitations culturelles au sein d'ARTE, ont miné cette croyance en l'omnipotence de la télévision. Mais cette croyance est remplacée par une autre: l'avènement d'une société de l'information. «L'évolution foudroyante des technologies de l'information et de la communication sera synonyme, pour l'Union européenne, d'une mutation économique et sociale si profonde qu'on peut déjà parler d'une troisième révolution industrielle [...] Le défi, dans le domaine social, sera d'offrir à tous les citoyens de l'Union un accès égal aux technologies de l'information " (Commission européenne, 1995, p. 3). Forte de cette certitude, la Commission multiplie les banques de données, les serveurs Internet et autres CD ROM. Or, non seulement cette politique s'appuie sur un déterminisme technologique simpliste (Wolton, 1999), mais, en plus, elle est en totale inadéquation avec la demande des citoyens européens: les supports d'information préférés de ces derniers sont, dans l'ordre, la télévision, la presse, les brochures et la radio ; Internet et le CD-ROM n'arrivent qu'en huitième et neuvième position ${ }^{5}$. De même, comme le rappellent fort à propos Pierre Bréchon et Grégory Derville, si le lien entre information et politisation paraît, dans l'Union européenne, scientifiquement établi, il n'existe pas de lien simple, mécanique, entre politisation et participation politique : «On peut, en effet, être fortement politisé et ne participer que faiblement à la vie politique, ou au contraire participer sans être politisé, comme on le voit fréquemment lors d'élection à forts enjeux» (Bréchon, Derville, 1997, p. 175). L'information n'entraîne pas automatiquement la participation politique. Surtout, trop d'information tue l'information. Multiplier les informations ne multiplie pas le nombre des personnes informées. Au contraire, une masse trop importante de données peut décourager et faire fuir le citoyen. En effet, l'homme a des capacités cognitives limitées et ne peut donner sens qu'à ce qu'il peut inscrire dans un cadre culturel et symbolique pré existant. Or, peu de citoyens ont une culture civique (connaissance des institutions européennes) et politique (maîtrise des enjeux européen) leur permettant de donner sens à l'information (électronique, télévisuelle ou papier) diffusée par les différents organes de la Commission. Par ailleurs, en voulant éviter de faire de la propagande, les différentes directions générales adoptent une démarche marketing de vente d'un produit. Démarche vouée à l'échec pour deux raisons principales. Premièrement, l'Europe n'est pas « un produit » fini, mais un chantier en construction, un projet politique en devenir. Deuxièmement, le marketing repose sur deux principes inadaptés à une communication politique à l'échelle de l'Union: la segmentation et la séduction. La segmentation consiste à choisir, dans le public potentiel, des cibles homogènes auxquelles on délivrera un message construit pour répondre aux attentes de la population visée. Or, si en matière de communication publique spécialisée, il convient d'identifier correctement les destinataires du message (par exemple, il est nécessaire que les informations concernant la politique agricole commune parviennent à tous les agriculteurs européens), il s'avère contre productif, en matière de communication politique européenne, de cibler telle ou telle catégorie du grand public. En effet, le problème de la Commission n'est pas de segmenter un marché unifié, mais de rassembler des populations hétérogènes pour former une Union politique 
européenne. Il ne s'agit donc pas de trouver des discriminants (ce qui distingue un cadre belge, d'un cadre italien), mais de rechercher des dénominateurs communs (ce qui rapproche un cadre suédois d'une fermière espagnole). De même, si la séduction fait partie intégrante de la communication politique, cette dernière ne peut, contrairement à ce que préconisait le rapport de Clerq (de Clerq, 1993), se résumer à la définition d'une image de marque attractive. Le débat, donc le dissensus, fait partie intégrante de la démocratie et de la communication politique. Il s'agit moins de séduire les citoyens européens, de les faire adhérer à un projet qui se construit sans eux, que de les intéresser et de les inviter à participer à la construction européenne. Or, on l'a vu lors du Traité de Maastricht et lors du passage à l'Euro, les institutions européennes insistent plus sur la nécessité de faire l'Union, que sur le désir de vivre ensemble. L'utopie européenne se transforme alors en un monstre froid, un mal nécessaire, synonyme de règlements tatillons et de jungle économique.

\section{Les problèmes structurels d'une communication politique à l'échelle de l'Union}

Cependant, au-delà de ces erreurs tactiques et stratégiques circonstancielles, l'inefficacité de la politique de communication de la Commission s'explique par des raisons plus profondes. En effet, la politique de communication de l'Union européenne se heurte à quatre types de problèmes structurels.

6 - Des problèmes opérationnels. Les fonctionnaires européens en charge de la communication n'ont pas un travail facile, puisque le public auquel ils sont confrontés est plurilingue, nombreux ( 370 millions d'habitants) et vit dans quinze pays géographiquement et culturellement très différents. De plus, ils doivent transmettre une information technique qui, d'une part, est souvent écrite dans un "eurojargon" inaccessible au commun des mortels et qui, d'autre part, croit de manière exponentielle au fur et à mesure des traités marquant l'élargissement et l'approfondissement de l'Union. Dans ces conditions, il est difficile de transmettre l'information directement au citoyen européen, d'autant plus que les réseaux animés par la DGX et ceux créés par les autres organes européens ${ }^{6}$ sont parfois en position de concurrence, plutôt qu'en situation de complémentarité. Par ailleurs, au vu du principe de subsidiarité, ce sont les Etats qui devraient prendre en charge l'information européenne de leurs concitoyens. Or, beaucoup rechignent à le faire, ou mettent en œuvre des campagnes donnant plus un point de vue national qu'une vision européenne des enjeux.

7 - Des problèmes financiers et humains. Le budget et le personnel dont dispose la DG X est largement inférieur à celui d'une agence de publicité de dimension européenne. De plus, il n'existe pas, au sein de la Commission, une véritable culture de la communication. Les quelques deux cents personnes qui, au siège des directions générales, sont chargées de la communication et, à ce titre, sont les correspondants privilégiés de la DG X, ne sont pas toutes intégrées dans une unité "information" ou "communication» spécialisée et manquent donc singulièrement de moyens logistiques pour mener leurs tâches à bien. Surtout, elles n'ont pas forcément la formation initiale ou l'expérience professionnelle nécessaire pour assumer au mieux leurs fonctions : il n'existe pas de concours spécifique pour devenir responsable de la communication dans une direction générale de la Commission. Enfin, si la DG X possède une unité consacrée à l'étude de l'opinion, elle ne 
possède pas d'unité spécialisée dans l'évaluation des campagnes ce qui permettrait d'en mesurer l'efficacité et surtout d'en améliorer progressivement le contenu. Il faut dire que la plupart des lignes budgétaires consacrées à des actions de communication prévoient parfois des financements pour des évaluations d'étape (6 mois dans le cadre de Citizen First), mais pratiquement jamais pour une évaluation finale.

8 - Des problèmes organisationnels : Il existe des tensions entre les directions générales et la DG X. En effet, certaines DG n'ont pas de budget communication propre, la ligne de crédit correspondant étant gérée par la $D G X$; tandis que d'autres DG gèrent directement les sommes consacrées à la communication. Ainsi, dans le programme Prince, c'était la DG XV qui exécutait le budget prévu pour la campagne "Citizen First", or dans le programme Prince II, la ligne budgétaire qui pourra être utilisée par la DG XV (Dialogue permanent avec les citoyens et les entreprises) est gérée par la DG X. Ces tensions internes à la Commission se doublent de tensions institutionnelles entre le Parlement européen et la DG X. Le premier reproche à la seconde son inefficacité chronique, tandis que la seconde reproche au premier de vouloir la transformer en organe de propagande au service exclusif de la réélection des députés. Tensions qui se déroulent dans un contexte où la Commission se voit fortement remise en cause. La Cour des comptes a, en 1997, relevé de très nombreuses irrégularités dans l'utilisation des subventions communautaires (environ 4 milliards d'Euros, soit $5 \%$ des dépenses totales). Rapport qui a donné lieu à des enquêtes qui ont abouti à la démission de la Commission Santer. Mais, comme le rappellent les actes du séminaire «Comment gérer l'Europe en l'an 2020 », la crise dépasse largement le cadre des malversations financières, puisque ce texte met en avant: des règles et des procédures lourdes et sclérosantes, une pyramide hiérarchique paralysante, une mauvaise gestion des ressource humaines, l'opacité du circuit de décision, l'absence de canaux vers la société civile, etc. (Prometheus Europe, 1999).

- Des problèmes politiques. Ce n'est sans doute pas un hasard si tous les rapports officiels se penchant sur la politique de communication de l'Union européenne mettent en avant la faiblesse politique de celle-ci. Cette faiblesse est due : à son incapacité à construire une Europe sociale et politique (Oostlander, 1993, p. 12), à son impuissance politique dans les affaires du monde (Oostlander, 1993, p. 5), à l'opacité et à la complexité de ses procédures de décisions (Pex, 1998, p. 6 ; Oostlander, 1993, p. 3), à des promesses faites qui n'ont pas été tenues en temps voulu (De Clerq, 1993, p.6) et à la disparition d'un «ennemi politique » qui faisait de l'Union européenne une nécessité vitale (De Clerq, 1993, p. 1). De plus, le Parlement européen explique la faiblesse politique de l'Europe, par le manque de pouvoir accordé à la seule institution européenne élue au suffrage universel et par l'absence de participation des citoyens et de la société civile à la construction de l'Europe politique (Parlement européen, 1996). Le juriste Dominique Rousseau avance une explication complémentaire : «en multipliant les institutions, en s'entassant les uns sur les autres, en se modifiant les uns les autres, traités et conventions ont donné naissance à un « monstre » constitutionnel incompréhensible par les citoyens et, peut-être même, par les initiés»(Rousseau, 1999, p.20). D'autres analystes soulignent l'absence d'une «euroclasse politique » ainsi que l'ambiguïté du principe de subsidiarité (Prometheus Europe, 1999) ou rappellent l'analyse de Raymond Aron selon laquelle «L'erreur des pères fondateurs de l'Europe est d'avoir méconnu la distinction hégélienne, reprise par Marx, du citoyen et du sujet économique » (Aron, cité par Zimmmer, 1999, p. 22). Enfin, comme le rapporte Juliette Bridier (Bridier, 1999), la Commission elle-même reconnaît que le concept ambitieux de citoyenneté européenne introduit dans les traités de l'Union ne «se 
traduit pas encore par des dispositions qui confèrent des droits effectifs [..]» (Commission européenne, 1995b). L'importance des problèmes politiques soulevés montre que l'on confond peut-être le symptôme avec la maladie. Si l'Union européenne souffre bel et bien d'un déficit de communication, celui-ci est sans doute la résultante, et non la cause, d'une grave dépression politique qui mine en profondeur l'Europe des quinze.

\section{Une démocratie européenne en quête d'espace public}

L'Union européenne traverse une profonde crise politique. La construction européenne est en panne faute d'une entente entre les Etats sur les réformes institutionnelles à mettre en place avant l'élargissement, mais surtout, et plus profondément, faute d'un projet politique qui soit soutenu par les citoyens des pays membres. «Or, la question de l'Europe politique est celle du sens, des perspectives historiques que les peuples voudront bien se donner, des valeurs sur lesquelles les Européens entendent fonder leur «Communauté de destin » (Zimmer, 1999, p. 23). On l'aura compris, la crise politique des institutions européennes renvoie à l'identité même de l'Europe. La chute du mur de Berlin a entraîné la disparition d'un contre modèle politique unificateur qui était la composante essentielle de l'identité négative de l'Union' (Cette chute a engendré une recomposition complète de la carte européenne. Cette dernière fut source de nombreux conflits armés (Bosnie, Arménie, Tchétchénie, etc.) qui posent, on la vu lors du conflit du Kosovo, la question d'une politique de défense commune qui, malgré la volonté de pays comme la France et l'Italie, reste dans l'ombre tutélaire de l'OTAN. De plus, cette instabilité aux frontières de l'Union européenne provoque de graves tensions entre partisans d'un élargissement immédiat (Allemagne, notamment) et ceux d'un approfondissement préalable (pays du sud de l'Europe). Surtout, l'effondrement du bloc communiste remet au goût du jour les sempiternelles querelles idéologiques autour $\mathrm{du}$ futur visage de l'Europe : fédération? confédération? ou simple zone de libre échange? Ni le Traité de Maastricht (1993) ni celui d'Amsterdam (1997) n'ont su trancher. Les élites européennes veulent construire une maison commune sans avoir défini les plans du bâtiment, ce qui ne peut que nourrir la méfiance des futurs propriétaires : les citoyens de l'Union. Or, ce problème identitaire se cristallise à une époque où les citoyens européens perdent confiance dans le politique. En effet, la panne de l'Europe politique survient au moment même où la globalisation des marchés et la mondialisation des technologies de communication viennent déstabiliser les cadres de références individuels, bouleverser l'organisation du travail au sein de l'entreprise et limiter considérablement les marges de manœuvre des différents gouvernements européens. Or, ces derniers doivent faire face à une distension dramatique du lien social et ne parviennent pas à enrayer l'exclusion ${ }^{8}$ (, ce qui ne fait qu'accroître la crise de la représentation (Rosanvallon, 1998) et génère ce que Pierre Calarne nomme l'acratie: "la conscience de devoir changer et le sentiment d'impuissance à le faire ou à trouver en soi la volonté de le faire »(Calame, 1995, p. 23). Crise identitaire de l'Union et acratie se conjuguent et se traduisent par une perte de sens et la montée corrélative d'un sentiment de défiance vis-à-vis de la construction européenne. Ce sentiment grandit au fur et à mesure que les politiques nationales et européennes proposent des recettes technocratiques concoctées par une élite de fonctionnaires dévoués mais qui manquent de légitimité démocratique. En effet, contrairement aux administrations nationales, les instances européennes ne peuvent appuyer leurs décisions sur l'existence d'un espace démocratique populaire où sont 
proposées, critiquées et amendées les grandes décisions politiques. De leur côté, les acteurs sociaux favorables à la construction européenne ne cessent de réclamer un «dialogue civil» que les instances européennes ne parviennent pas à mettre en place ${ }^{9}$ (Autrement dit, comme le rappelle Dominique Wolton, ni les éléments de la société civile favorables à l'Europe politique ni les élites administratives européennes ne peuvent s'appuyer sur un espace public large et populaire qui assoirait la légitimité démocratique du projet politique européen (Wolton, 1993). Or, l'espace public est, tout à la fois, le lieu des cohabitations culturelles (Hermès, 1999), de la construction d'un monde commun (Tassin, 1991), de l'affrontement social (Floris, 1995) et, dans une perspective kantienne que nous ferons nôtre, le lieu de contrôle et de légitimation du politique. L'espace public, dans sa dimension politique, est l'espace du débat démocratique (débat alimenté par la publicité de l'information gouvernementale), mais aussi le lieu de l'action politique. Cet espace public peut s'incarner dans des lieux physiques (l'agora athénienne ou l'Assemblée nationale) mais possède également une forte dimension symbolique. À l'heure actuelle, l'espace public est national (et/ou local) et fortement structuré par les mass médias (Ferry, 1989). Or, l'Union européenne est un espace transnational qui ne possède pas de mass média unificateur permettant à la communauté des citoyens de l'Union de se rendre visible à elle-même. Certes, il existe, dans l'Union européenne, un certain nombre de lieux où se développent des débats européens sur des questions de politique européenne comme le Parlement européen, l'Institut universitaire de Florence ou les Conférences ínter citoyennes ${ }^{10}$ (Mais ces lieux restent réservés à une élite très restreinte, la plupart du temps, on l'a vu encore aux dernières élections européennes, les questions européennes restent abordées sous un angle national ou régional.

\section{Emergence d'un espace public européen : différents scénarios}

11 Pourtant, théoriquement, rien ne s'oppose à ce que naisse, un jour, un espace public européen. C'est, en tout cas, la thèse que défend Jürgen Habermas. Prenant acte de l'effondrement de l'Etat-nation, mais reconnaissant la vitalité de la société civile européenne, il prédit l'avènement d'une démocratie délibérative où l'espace public européen se fonderait sur une identité politique, "un patriotisme constitutionnel», distinct de l'identité culturelle : « Les opinion publiques nationales sont encore largement isolées culturellement les unes à l'égard des autres. [...] Dans l'avenir toutefois, on pourrait assister à la différentiation des cultures nationales d'une part et d'une culture politique d'autre part [...]. À la différence des Etats-Unis, un patriotisme constitutionnel européen doit se relier à des principes juridiques universalistes uniques à partir de perspectives différentes imprégnées par l'histoire nationale » (Habermas, 1992, pp 37-38). Loin de cette vision normative, la Commission européenne, à travers son projet de société de l'information, semble plutôt parier sur les nouvelles technologies pour rapprocher les citoyens des instances européennes. En filigrane, on retrouve l'idée d'un espace public virtuel, qui via Internet viendrait pallier le déclin d'une démocratie représentative noté par certains intellectuels (Rosanvallon, 1998; Touraine, 1989). Or, tout un ensemble d'études empiriques sur l'impact des nouvelles technologies sur la participation politique (Dacheux, 1998; Massit Folléa, 1996; Monnoyer, Maigret, 1998; O’Donnell, Trench, Ennals, 1998) montrent que les NTIC en général, et Internet en particulier, n'élargissent pas le cercle des citoyens participant à la vie politique. Les NTIC ne créent pas un nouvel 
espace public, mais renforcent les capacités d'action des citoyens organisés collectivement, sous forme associative notamment. Dès lors, entre un scénario politique pariant, sans doute trop vite, sur la formation d'une «identité postnationale » (Ferry, 1992) et un projet technologique enterrant trop rapidement les organisations sociales, il est possible d'entrevoir un autre scénario. Celui-ci s'ancre dans un triple constat:

- les Etats-nations résistent (L'Etat n'est pas mort », rappelle fermement Henri Mendras ${ }^{11}$,

- les mouvements sociaux s'européanisent peu à peu (marche européenne contre le chômage, grève européenne des cheminots, etc.),

- les réseaux associatifs européens créent, à l'image des associations littéraires au Dixhuitième siècle, des espaces de délibérations publiques (Weisben, 1998).

Dès lors, on peut concevoir une troisième hypothèse, celle de l'émergence, lente et progressive, d'un espace public européen où l'Etat-nation reste fort, la représentation continue à jouer un rôle clef et où le développement des pratiques sociales transfrontalières (tourisme, travail, étude, etc.) et la multiplication des espaces internationaux de médiations (syndicats européens, ONG, médias, universités, etc.) permettent de dépasser la simple «cohabitation politique » actuelle au profit d'une interculturalité européenne, venant s'ajouter et non se substituer aux identités collectives existantes. L'espace public européen interculturel ne serait, dans cette perspective, qu'une complexification supplémentaire dans l'imbrication existante entre les différents espaces publics de l'Union européenne.

Le sens que nous donnons à l'adjectif «interculturel» inscrit notre propos dans une perspective théorique qui conçoit la culture comme étant le produit de la créativité humaine. Contrairement aux thèses culturalistes, la culture n'est pas un héritage collectif qui structurerait de manière uniforme tous les individus d'une même communauté. La culture n'est pas une essence, c'est une construction sociale permanente. Du coup, la notion «d'identité culturelle» ne peut devenir une dangereuse «illusion identitaire » (Bayart, 1996) que si elle est comprise comme étant une spécificité radicale servant à caractériser (dans les faits, à discriminer) des individus. Par contre, ce concept devient heuristique, dès lors que l'on défend une vision dynamique de la culture et de l'identité, le passage de l'un à l'autre se faisant par le biais de l'enculturation qui est « l'ensemble des processus conduisant à l'appropriation par l'individu de la culture de son groupe » (Camilléri, 1989, p. 28). Or, tout individu appartient à plusieurs groupes et, au cours de sa vie, change de nombreuses fois de milieux; c'est pourquoi l'identité culturelle de l'homme moderne est, forcément, plurielle. Dès lors, loin de signifier une uniformisation culturelle, la construction européenne peut devenir le moyen d'enrichir d'une nouvelle dimension l'identité collective de chaque habitant de l'Union. Mais le caractère plurinational de l'Europe repose, à nouveau, le problème qui nous a occupé, au début de ce texte: celui de la communication européenne. Comment les citoyens de l'Union désireux de profiter de cet enrichissement peuvent-ils surmonter leurs différences linguistiques et échapper à ces « langages silencieux» (Hall, 1973) qui font obstacles au dialogue entre individus de nationalités différentes? Bien entendu, il n'existe pas de réponse à une telle question. Pas de réponse théorique du moins. Cependant, nous pensons qu'une telle réponse peut surgir de l'étude des réseaux européens de citoyens qui, en leur sein, développent des communications politiques à l'échelle de l'Union. En effet, ces réseaux, qu'ils se nomment, «Helsinky's Citizen Assembly », " Eurotopia » ou 
«Mouvement européen international », nous semblent particulièrement importants, pour trois raisons :

17 - Ils diffusent, dans l'espace public, des textes politiques concernant l'Union européenne (pétitions pour une constitution européenne, appel pour une Europe sociale, etc.). Etudier les techniques mises en œuvre, les succès et les échecs de ces communications politiques devrait permettre d'envisager des solutions efficientes aux problèmes structurels que nous avons identifiés.

18 - Ils expérimentent, in vivo, l'Europe interculturelle qu'ils appellent de leurs vœux. Ils contribuent ainsi, en organisant des rencontres associatives européennes, à l'émergence d'espaces publics européens autonomes qui peuvent servir de points d'ancrage à un espace public européen non institutionnel. Saisir les contours de ces espaces publics européens autonomes et éphémères, peut permettre de mieux appréhender le processus de construction de l'espace public européen.

19 - Ce sont des médiateurs. D'une part, ils permettent à l'ensemble du monde associatif de prendre conscience des enjeux européens comme on l'a vu, en France, avec l'appel "Refondons l'Europe» signé par des organisations jusqu'ici peu sensibilisées aux questions européennes ${ }^{12}$. D'autre part, ils initient les citoyens aux enjeux européens en prenant part aux campagnes d'informations européennes et font remonter certaines attentes de la société civile vers certaines directions générales qui les associent étroitement à la définition des politiques publiques européennes (c'est le cas, notamment, de la direction générale chargée de l'environnement). Etudier ces différents types de médiation offrirait une meilleure connaissance des réseaux sociaux actifs dans l'Union européenne et permettrait de réajuster une politique de communication européenne qui oscille entre la séduction marketing et la fascination technologique.

20 Le désir d'Europe est en panne. Or, si la communication de l'Union européenne est, sans conteste, perfectible, il ne faut pas voir dans ses faiblesses actuelles la raison principale de l'euroscepticisme. En réalité, les problèmes de communication que rencontre l'Union européenne ne sont que les symptômes d'un malaise plus profond. Face à la globalisation économique et aux fortes traditions culturelles de chacun des pays membres, les élites européennes ne parviennent pas à formuler des réponses politiques permettant de mettre fin à la crise identitaire de l'Union. Dès lors, elles ont la tentation de proposer une réponse technologique à des problèmes politiques qu'elles ne peuvent résoudre seules. C'est pourquoi, elles cherchent à pallier l'absence d'espace public européen par la mise en place de réseaux électroniques. Pourtant, l'histoire politique des pays comme la France, l'Allemagne ou la Grande-Bretagne montre que ce ne sont pas des réseaux techniques, mais des réseaux sociaux et, en particulier, les associations qui ont pu favoriser l'émergence d'un espace public venant légitimer et asseoir durablement la démocratie (Eley, 1992). Loin d'accélérer la fin de la représentation, la participation des citoyens est, aujourd'hui, le plus sûr moyen de la préserver. 


\section{BIBLIOGRAPHIE}

ARENDT H., La condition de l'homme moderne, Paris, Calman-Levy, 1983. Bayart P., L'Illusion

identitaire, Paris, Fayard, 1996.

BRECHON P., DERVILLE G., "Politisation et exposition à l'information », in P. BRECHON, B.

CAUTRES, Les enquêtes eurobaromètres, Paris, L'Harmattan, 1997.

BRIDIER J., « La citoyenneté européenne : un projet de cohabitation culturelle », Hermès, N$^{\circ}$ 23/24, 1999.

CALAME P., « La citoyenneté en Europe », Territoires, N 354, 1995.

CAMILLERI C, Chocs de cultures, Paris, L'Harmattan, 1989.

Commission européenne, Rapport de synthèse du Forum européen sur la politique sociale 1998, Luxembourg, Office des publications officielles des Communautés européennes, 1999.

Commission européenne, La société de l'information, L'Europe en mouvement, DG X, 1995.

Commission européenne, Rapport de la commission pour le groupe de réflexion sur la CIG, Bruxelles, 1995 b.

Commission européenne, Croissance, compétitivité, emploi, Bruxelles, Commission européenne, 1994.

Commission européenne, Livre vert sur la télévision sans frontières, Bruxelles, Commission européenne, 1984.

CLERQ W. de, Réflexion sur la politique d'information et de communication de la Communauté européenne, Bruxelles, Commission européenne, 1993. Dacheux E., « Un monde méconnu : les réseaux associatifs européens », Hermès, N²3/24, 1999.

DACHEUX E., « Internet, un outil susceptible de réveiller le militantisme des citoyens européens? ", Metz, Actes du onzième congrès des sciences de l'information et de la communication, 1998.

ELEY G, « Nation, Publics and Political Cultures », in C. Calhoun, Habermas and the Public Sphere, Cambridge (Mass.), The MIT Press, 1992.

FERRY J-M., « Les transformations de la publicité politique », Hermès, N 4, 1989.

FERRY J-M., « Pertinence du postnational », in J. Lenoble, N. Dewandre, l'Europe au soir du siècle, Paris, Éditions Esprit, 1992.

FLORIS B., «L'entreprise sous l'angle de l'espace public », in I. Paillart, L'espace public et l'emprise de la communication, Grenoble, Ellug, 1995.

HABERMAS J., «Citoyenneté et identité nationale » in J. Lenoble, N. Dewandre, l'Europe au soir du siècle, Paris, Éditions Esprit, 1992.

HABERMAS J., L'espace public, Paris, Payot, 1990.

HALL E.T., Le langage silencieux, Paris, Seuil, 1973.

HERMES, Les cohabitations culturelles en Europe, Paris, Éditions du CNRS, 1999. 
KIM Y.Y., GUDYKUNST W.B., Theory in Intercultural Communication, London, Sage, 1988.

Laidi Z., « La mondialisation ou la radicalisation de l'incertitude », Etudes, mars, 1997.

LIPIANSKI, E-M., « Groupes et identité » in G. Michaud, Identités collectives et relations interculturelles, Bruxelles, Edition Complexe, 1978.

MASSIT-FOLLEA F., « La démocratie électronique » in J. Mouchon, F. Massit-Folléa, Information et démocratie, Fontenay St-Sloud, ENS Éditions, 1997.

MENDRAS H., « Le mal de Bodin », Le Débat, № 105, 1999.

MONNOYER L., MAIGRET E., Télévision interactive et démocratie locale, Paris, Rapport d'étude du laboratoire «Communication et Politique », CNRS, 1998.

O’DONNELL S., TRENCH B., ENNALS K., Weak Connections, Dublin, Dublin City University, 1998.

OOSTLANDER A., Rapport sur la politique d'information de la Communauté, Strasbourg, Parlement européen, 1993.

Parlement européen, Résolution sur la participation des citoyens et des acteurs sociaux au système institutionnel de l'Union européenne, Strasbourg, Journal officiel des communautés européennes, 1996.

Parlement européen, Résolution sur la politique d'information de la Communauté, Strasbourg, Journal officiel des communautés européennes, 1993.

PEX P., Rapport sur la politique d'information et de communication dans l'Union européenne, Strasbourg, Parlement européen, 1998.

Prometheus-Europe, Comment gérer l'Union européenne en 2020 ?, Paris, Centre Kléber, 1999.

ROSANVALLON P., Le nouveau travail de la représentation, Esprit, février, 1998.

ROUSSEAU D., « Pour une constitution européenne », Transversales, N 56, 1999.

TASSIN E., « Espace commun ou espace public ? », Hermès, N 10, 1991.

TOURAINE A., « Communication politique et crise de la représentativité », Hermès, N 4, 1989.

WEISBEN J., « Construire la citoyenneté européenne », Communication au colloque Action collective et intégration européenne, Oxford, 1998.

WOLTON D., La dernière utopie, Paris, Flammarion, 1993.

WOLTON D., Internet et après ?, Paris, Flammarion, 1999.

ZIMMER A.-C, « La citoyenneté pour entrer dans l'Europe politique », Transversales, N 56, 1999.

\section{NOTES}

1. La réforme de la politique de communication de la Commission lancée par le nouveau président de la Commission n'est pas encor e complètement achevée, mais elle s'est traduite, entre autre, par la disparition de la DG X dont certaines missions sont aujourd'hui gérées par la Commissaire chargée "de l'éducation et de la culture, de l'Office des publications, de l'Europe des citoyens et de la transparence", Viviane Reding.

2. Tous les chiffres cités dans ce paragraphe concernent l'année 1998.

3. Cet office publie environ 1500 titres par an, chiffre qui doit être complété par les quelques 200 titres que les diverses institutions de l'Union européenne publient par leurs propres moyens. 
4. La Commission européenne encourage les universitaires européens à développer des enseignements sur la construction européenne en finançant des chaires Jean Monnet.

5. Source : Eurobaromètre, $\mathrm{N}^{\circ} 49,1998$. L'Eurobaromètre n'est pas un instrument capable de rendre compte de l'opinion publique européenne, mais de quinze opinons nationales. Cependant, il donne parfois des indications utiles sur les pratiques et les désirs des citoyens de l'Union.

6. Parmi les plus connus, on peut citer, les Euro-Info-Centres (DG XX III), les Business Innovation Centre (DG XVI) et les data shop (Eurostat).

7. E.M Lipianski (Lipianski, 1978) rappelle que toute identité collective met en œuvre une identité positive (celle que le groupe se propose comme modèle) et une identité négative (les traits dans lesquels le groupe ne veut pas se reconnaître).

8. Même si les chiffres suivants ne sont que des estimations sujettes à controverse, ils donnent une idée de l'ampleur du phénomène: l'Union européenne comporte plus de 1,5 millions de personnes survivant sans abris, et plus de 60 millions de personnes vivant en dessous du seuil de pauvreté (fixé, en général, à $50 \%$ de la moyenne nationale du PIB par habitant).

9. Par exemple, la Commission n'a su organiser que deux «Forum européen sur la politique sociale ", manifestation rassemblant 1500 représentants d'ONG, de syndicats, de gouvernements et de la Commission afin de débattre des questions clefs de la politique sociale européenne. Le dernier en date (juin 1998) ne pouvait que constater le fait suivant: «un dialogue a été établi entre le $\mathrm{ONG}$, la Commission et le parlement européen dans le cadre des travaux préparatoires à la conférence inter-gouvemementale de 1996-1997 en vue de la modification des traités. Au nombre des questions soulevées figurait l'établissement, dans le traité, de bases pour le dialogue civil avec les ONG. Toutefois, comme nous l'avons constaté, ces modifications n'ont pas été introduites dans le traité d'Amsterdam (...) ». (Commission européenne, 1998, p. 8).

10. Les conférences inter-citoyennes (ou $\mathrm{CIC}$ ) réunissent, tous les six mois, des militants européens appartenant à une trentaine de réseaux associatifs militant pour une démocratie européenne plus participative.

11. Henri Mèndras, « Le mal de Bodin », Le débat, $\mathrm{N}^{\circ} 105,1999$.

12. Cet appel fut diffusé le 6 novembre 1997. Il est signé aussi bien par des militants associatifs européens comme Robert Toulemon ou des députés européens comme Pervenche Berès, mais aussi par des représentants d'associations françaises oeuvrant dans le domaine social (René Lenoir pour l'UNIOPSS), des droits de l'homme (Henri Leclerc pour le Ligue des droits de l'homme) ou de la réflexion sur la vie associative (Frédéric Pascal pour la Fonda).

\section{RÉSUMÉS}

Les forts taux d'abstention aux dernières élections européennes, montrent qu'une large partie des européens se montre circonspecte voire méfiante vis-à-vis de la construction européenne. Certains attribuent cet "euroscepticisme » à une défiance de la politique de communication de l'Union européenne. Note article cherche à cerner la part de vérité, mais aussi la part de «fausse évidence » de cette affirmation. Pour ce faire, nous avons procédé à une analyse critique la politique de communication mise en place par la Commission européenne (avant l'arrivée de Romano Prodi), puis mis en lumière l'absence d'un espace public européen.

The high abstention rates at the last European elections show that many Europeans are circumspect or even distrustful of the European «construction». Some attribute this 
" euroscepticism» to a defiance of the European union's communications policy. Our article seeks to measure not only the validity of this affirmation, but also the extent of « false evidence

« therein.

INDEX

Mots-clés : communication politique, espace public européen, Commission européenne, Europe politique

\section{AUTEUR}

\section{ÉRIC DACHEUX}

Éric Dacheux, maître de conférences à l'IUT de Roanne, vient de publier « Association et communication : critique du marketing ", CNRS Éditions, 1998 et a coordonné le numéro 23/24 de la revue Hermès intitulé « Les cohabitations culturelles en Europe ». Il anime, au sein du laboratoire CNRS « Communication et politique », une équipe de six chercheurs travaillant sur l'espace public européen. IUT de Roanne, Laboratoire CNRS «Communication et Politique» 